\title{
ARTICLE
}

Cellular and Molecular Biology

\section{The anaphase-promoting complex/cyclosome: a new promising target in diffuse large B-cell lymphoma and mantle cell lymphoma}

Anke Maes ${ }^{1}$, Ken Maes ${ }^{1}$, Hendrik De Raeve ${ }^{2}$, Eva De Smedt ${ }^{1}$, Philip Vlummens ${ }^{1,3}$, Vanessa Szablewski ${ }^{4}$, Julie Devin ${ }^{5}$, Sylvia Faict ${ }^{1}$, Kim De Veirman ${ }^{1}$, Eline Menu ${ }^{1}$, Fritz Offner ${ }^{3}$, Marcel Spaargaren ${ }^{6}$, Jérôme Moreaux ${ }^{5}$, Karin Vanderkerken ${ }^{1}$, Els Van Valckenborgh ${ }^{1}$ and Elke De Bruyne ${ }^{1}$

\begin{abstract}
BACKGROUND: The aggressive B-cell non-Hodgkin lymphomas diffuse large B-cell lymphoma (DLBCL) and mantle cell lymphoma $(\mathrm{MCL})$ are characterised by a high proliferation rate. The anaphase-promoting complex/cyclosome (APC/C) and its co-activators Cdc20 and Cdh1 represent an important checkpoint in mitosis. Here, the role of the APC/C and its co-activators is examined in DLBCL and MCL.

METHODS: The expression and prognostic value of $\mathrm{Cdc} 20$ and Cdh1 was investigated using GEP data and immunohistochemistry. Moreover, the therapeutic potential of APC/C targeting was evaluated using the small-molecule inhibitor proTAME and the underlying mechanisms of action were investigated by western blot.

RESULTS: We demonstrated that Cdc20 is highly expressed in DLBCL and aggressive $M C L$, correlating with a poor prognosis in DLBCL. ProTAME induced a prolonged metaphase, resulting in accumulation of the APC/C-Cdc20 substrate cyclin B1, inactivation/ degradation of $\mathrm{BCl}-2$ and $\mathrm{BCl}-\mathrm{xL}$ and caspase-dependent apoptosis. In addition, proTAME strongly enhanced the anti-lymphoma effect of the clinically relevant agents doxorubicin and venetoclax.

CONCLUSION: We identified for the first time APC/C as a new, promising target in DLBCL and MCL. Moreover, we provide evidence that Cdc20 might be a novel, independent prognostic factor in DLBCL and MCL.
\end{abstract}

British Journal of Cancer (2019) 120:1137-1146; https://doi.org/10.1038/s41416-019-0471-0

\section{BACKGROUND}

Diffuse large B-cell lymphoma (DLBCL) and mantle cell lymphoma $(\mathrm{MCL})$ are among the most common aggressive B-cell non-Hodgkin lymphomas (NHL). Although the standardof-care regimen R-CHOP (rituximab, cyclophosphamide, doxorubicin, vincristine and prednisone) has significantly improved the survival rates, MCL remains incurable and up to one-third of the DLBCL patients are or become refractory. ${ }^{1,2}$ Within the DLBCL patients, two major DLBCL subtypes are identified by gene expression profiling: germinal centre $B$ cell (GCB) and activated $B$-cell $(A B C) D L B C L$, of which the $A B C-D L B C L$ subtype has a worse clinical outcome. ${ }^{3}$

The clinical use of cell cycle targeting agents, such as the microtubule-targeting agent vincristine, has proven that this is an interesting approach in the treatment of high-grade B-cell $\mathrm{NHL}^{4}$ However, these agents induce severe toxicity and are associated with multidrug resistance development. ${ }^{5}$ Clinical studies are currently ongoing to find more selective cell cycle targets by blocking either the interphase (e.g. Cdk inhibitors) or mitotic entry (e.g. microtubule-targeting agents, Plk-1 and aurora kinase inhibitors). ${ }^{6}$ In B-cell $\mathrm{NHL}$, the anti-lymphoma effects of these agents were either disappointing or the trials were suspended early due to toxicity issues. ${ }^{6-9}$ Recent preclinical studies show that targeting molecules involved in the mitotic exit, such as the anaphase-promoting complex/cyclosome (APC/C), is a better strategy since it provokes a more permanent mitotic arrest, thereby reducing the chance of mitotic slippage and thus enhancing mitotic cell death. ${ }^{10}$ The activation of the APC/C, an E3-ubiquitin ligase, depends on the interaction with its coactivators Cdc20 or Cdh1. The spindle assembly checkpoint (SAC) prevents APC/C activation in early mitosis. When biorientation of the chromosomes is achieved in the metaphase, the SAC pathway is inactivated and APC/C-Cdc20 binding occurs. This interaction leads to proteasomal degradation of the substrates cyclin B1 and securin, subsequently allowing the onset of the anaphase. ${ }^{11}$ Cdc20 is thereafter replaced by $\mathrm{Cdh} 1$ and the APC/C-Cdh1 targets Cdc20, aurora kinases and Plk-1 during mitotic exit. In early $\mathrm{G} 1$ phase, Skp2 and mitotic cyclins are degraded, resulting in increased p21, p27 and cyclin D levels, which is necessary to maintain the G1 phase. ${ }^{11,12}$

\footnotetext{
${ }^{1}$ Department of Hematology and Immunology, Myeloma Center Brussels, Vrije Universiteit Brussel, Brussels, Belgium; ${ }^{2}$ Department of Pathology, UZ Brussel, Vrije Universiteit Brussel, Brussels, Belgium; ${ }^{3}$ Hematology, Department of Internal Medicine, Ghent University Hospital, Ghent, Belgium; ${ }^{4}$ Department of Biopathology, CHU Montpellier,

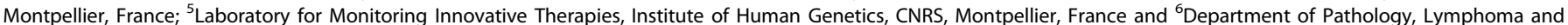
Myeloma Center Amsterdam (LYMMCARE), Cancer Center Amsterdam, Amsterdam UMC, University of Amsterdam, Amsterdam, the Netherlands Correspondence: Elke De Bruyne (Elke.De.Bruyne@vub.be)
} 
Several studies have already suggested the involvement of the APC/C and its co-activators in tumorigenesis of different cancers and their potential as a new therapeutic target. ${ }^{11,13}$ Wang et al. were the first to demonstrate APC/C mutations in human cancer cells, showing that a disruption of some of the APC/C subunits contributes to tumorigenesis by deregulation of key cell cycle regulators. ${ }^{14}$ Increased Cdc20 expression is correlated with poor prognosis in several human cancers, ${ }^{15-21}$ and depletion of Cdc20 in various cancer cell lines resulted in tumour metaphase arrest and induction of apoptosis. ${ }^{19,22,23}$ Moreover, inhibition of Cdh1 has been associated with tumorigenesis and decreased Cdh1 expression is observed in breast and colon cancer. ${ }^{24,25}$ The small molecule TAME (tosyl-L-arginine methyl ester) was discovered as a specific APC/C inhibitor in the past decade. It mimics the IR-tail of the co-activators and blocks the interaction between APC/C and Cdc20 or Cdh1. ${ }^{26}$ Previously, we and others demonstrated that proTAME (permeable variant of TAME) treatment results in a reduced viability, a growth arrest and apoptosis of malignant plasma cells. $^{15,27}$

Currently, however, little is known about the therapeutic potential of targeting the APC/C and its co-activators in DLBCL and MCL. The aim of this study is to investigate the expression of the APC/C co-activators $\mathrm{Cdc} 20$ and $\mathrm{Cdh} 1$ and the therapeutic potential of $A P C / C$ targeting in $\mathrm{DLBCL}$ and $\mathrm{MCL}$.

\section{METHODS}

Analysis of Cdc20 and Cdh1 gene expression levels

The publicly available GEP datasets GSE10846 ${ }^{28,29}$ (containing gene expression data and survival data of 167 ABC-DLBCL patients, $183 \mathrm{GCB}-\mathrm{DLBCL}$ patients and 64 unclassified DLBCL patients), GSE56315 $5^{30}$ (containing gene expression data of $33 \mathrm{~B}$ cell samples, $23 \mathrm{ABC}-\mathrm{DLBCL}$ patients, $29 \mathrm{GCB}-\mathrm{DLBCL}$ patients and 3 unclassified DLBDL patients), GSE16455 $5^{31}$ (containing gene expression data of 7 indolent and 15 aggressive MCL patients) and GSE36133 32 (containing gene expression data of 13 human DLBCL cell lines and 5 MCL cell lines) were used. Raw CEL files were obtained from the Gene Expression Omnibus (GEO) and gcrma-normalisation was performed in $\mathrm{R}$ using bioconductor. Survival analysis of GEP microarray data was done using Genomicscape (http://genomicscape.com). The following probe sets were used: 202870-s-at (Cdc20) and 209416-s-at (Cdh1).

Patient biopsies and staining

Patient samples were collected at the Department of Pathology in Brussels (UZ Brussels, Belgium) and the Department of Biopathology in Montpellier (CHU Montpellier, France). Paraffin-embedded samples available from seven DLBCL patients were selected in Brussels and 3- $\mu \mathrm{m}$-thick sections from tissue microarrays containing three representative $0.6-\mathrm{mm}$ cores of routinely processed tissues from 27 DLBCL patients were included from Montpellier. ${ }^{33}$ All samples were stained with Cdc20 antibody (ATLAS Antibodies $A B$, Bromma, Sweden) using an automated immunostainer Benchmark XT (Roche Ventana, Basel, Switzerland). As a positive control, tonsils with secondary follicles were used. Two hundred tumour cells were counted and the percentage of tumour cells with a staining of any intensity was determined at a magnification $\times 400$ on a Leica DM2000 microscope. The counting was performed in hot-spot areas by means of an ocular grid. Based on the R-IPI of the DLBCL patients, they were divided into good and poor prognosis groups. Cells were scored MYC and $\mathrm{BCl}-2$ positive as we described previously. ${ }^{33}$

Cell culture

All the MCL (Jeko-1, Mino and Rec-1) and ABC-DLBCL cell lines (U2932 and RI-1) were maintained in the RPMI-1640 medium (Lonza, Basel, Switzerland) supplemented with $10 \%$ foetal calf serum (FCS) (Biochrom AG, Berlin, Germany) and $2 \mathrm{mM}$ glutamine (Life Technologies, Gent, Belgium). The GCB-DLBCL cell lines (SUDHL-6, OCl-Ly1 and OCl-Ly7) were maintained in the IMDM medium (Life Technologies) supplemented with 10\% FCS and 2 $\mathrm{mM}$ glutamine. Cells were cultured at $37{ }^{\circ} \mathrm{C}$ in a humidified $5 \%$ $\mathrm{CO}_{2}$ atmosphere. All cell lines were obtained from ATCC and regularly tested for mycoplasma contamination. They were authenticated by STR profiling.

\section{Reagents}

The APC/C inhibitor proTAME was obtained from R\&D Systems (Oxon, UK). Venetoclax and rituximab were obtained from Selleckchem (Bio-Connect, Huissen, The Netherlands), doxorubicin hydrochlorate was purchased from Sigma-Aldrich (Bornem, Belgium) and apcin was kindly provided by Dr. R.W. King (Department of Cell Biology, Harvard Medical School).

\section{Statistical analysis}

Prognostic significance of $\mathrm{Cdc} 20$ and $\mathrm{Cdh} 1$ gene expression was calculated using the MaxStat R package. Statistical differences in overall survival were calculated by a log-rank test and survival curves were plotted using Kaplan-Meier method. Multivariate analysis was performed using the Cox proportional hazards model. Graphical and statistical analysis was performed using GraphPad Prism 5.01 software. Statistical significance ( $p$-value of $p<0.05$ was considered significant) was determined by a Mann-Whitney U test (to compare two groups) and a one-way ANOVA with Bonferonni correction for multiple testing.

More details on the 'Methods' section are described in Supplemental Methods.

\section{RESULTS}

Cdc20 expression is increased in DLBCL and MCL patients and associated with poor survival

To investigate the clinical relevance of the APC/C in the aggressive B-cell malignancies DLBCL and MCL, the gene expression levels of the two APC/C co-activators, namely Cdc20 and Cdh1, were analysed using publicly available gene expression profiling (GEP) datasets. The expression of $\mathrm{Cdc} 20$ and Cdh1 mRNA was assessed in DLBCL patients, using GEP data from B-cell samples $(n=33)$, ABC-DLBCL $(n=190)$, GCB-DLBCL $(n=212)$ and unclassified DLBCL patients $(n=67)$. The gene expression levels of Cdc20 were significantly increased in all DLBCL subtypes compared with the B-cell samples. In contrast, the Cdh1 gene expression levels were not significantly different in the GCB-DLBCL and ABC-DLBCL patients compared with the B-cell samples (Fig. 1a, b). Analysis of gene expression levels in a MCL cohort revealed elevated Cdc20 expression levels in aggressive MCL compared with indolent MCL. No significant difference was observed for Cdh1 expression (Fig. 1c, d). Importantly, high Cdc20 expression levels were associated with a worse survival in DLBCL patients receiving the standard R-CHOP treatment. The same trend was observed for Cdh1 and clinical outcome; however, significance was not reached (Fig. 1e, f).

Next, the prognostic value of Cdc20 expression in DLBCL patients was compared with conventional prognostic factors, including the age at diagnosis, International Prognostic Index (IPI) and molecular DLBCL subtypes. Univariate COX analysis demonstrated that all these factors have a prognostic value in DLBCL (Table 1A). In the multivariate COX analysis, when all parameters were tested together, Cdc20 expression, IPI and molecular subtype remained independent prognostic factors (Table 1B). Finally, the protein $\mathrm{Cdc} 20$ expression was determined on DLBCL patient biopsies (Fig. 1g). The percentage Cdc20-positive lymphoma cells was counted and plotted against the patient's prognosis according to their R-IPI. A significant higher amount of Cdc20positive lymphoma cells was counted in the patients with a poor 

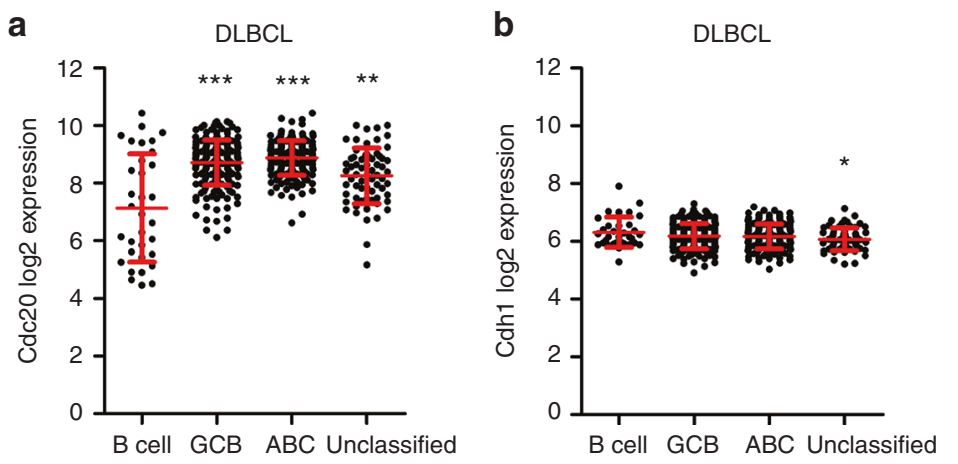

C

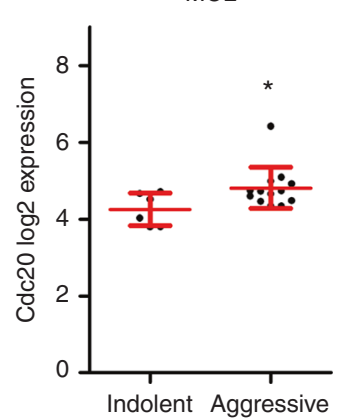

d

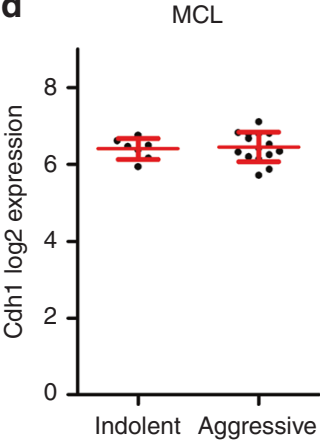

e

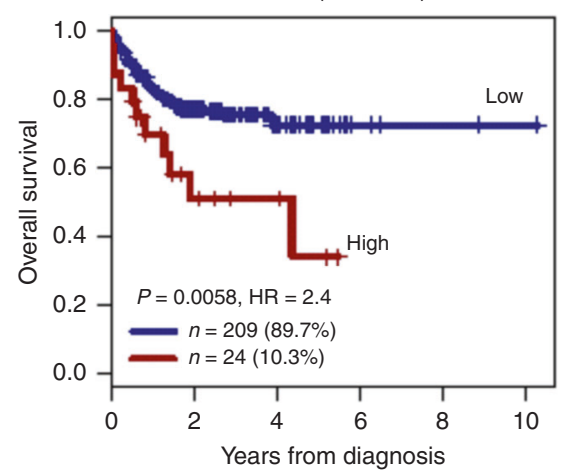

f

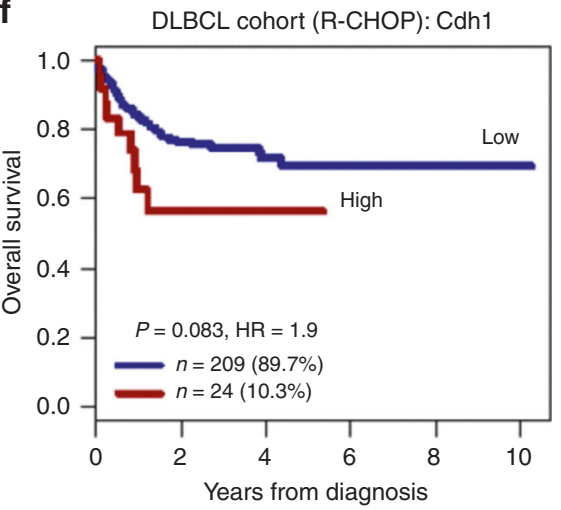

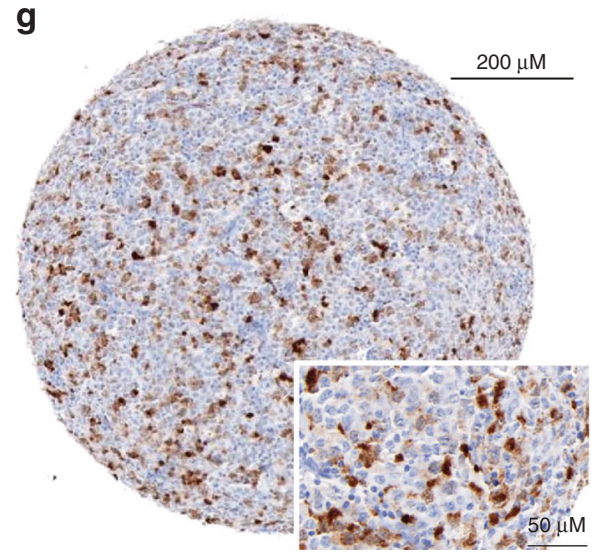

h

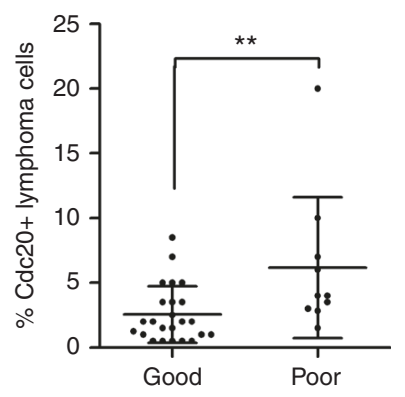

Prognosis according to R-IPI i

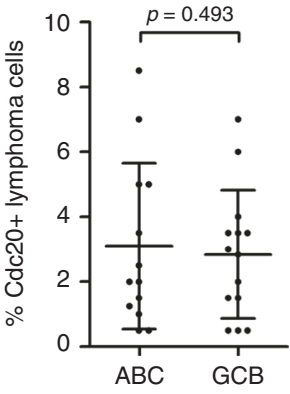

j

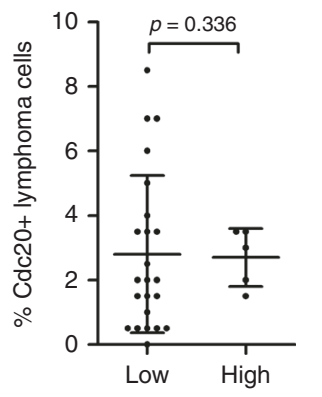

Bcl-2 + Myc expression

Fig. 1 Cdc20 mRNA expression is increased in DLBCL and MCL patients and is associated with worse survival in DLBCL patients. $\mathbf{a}$, $\mathbf{b}$ Cdc20 and Cdh1 mRNA expression in DLBCL. Cdc20 (a) and Cdh1 (b) gene expression levels of B-cell samples $(n=33)$, patients with ABC-DLBCL $(n=$ 190), patients with GCB-DLBCL $(n=212)$ and patients with unclassified DLBCL $(n=67)$ were obtained from the publicly available microarray datasets GSE10846 and GSE56315. Mean expression \pm SD is shown in red. ${ }^{* *} p<0.01$ and ${ }^{* * *} p<0.001$. c, d Cdc20 and Cdh1 mRNA expression in indolent and aggressive MCL. Cdc20 (c) and Cdh1 (d) gene expression levels of patients with indolent $(n=7)$ and aggressive MCL $(n=15)$ were obtained from the GSE16455 dataset. Mean expression \pm SD is shown in red. ${ }^{*} p<0.05$. e, f Prognostic value of Cdc20 and Cdh1 mRNA levels in terms of overall survival in DLBCL. The prognostic value of Cdc20 (e) and Cdh1 (f) was determined in DLBCL patients receiving RCHOP treatment from the Lenz cohort $(n=233)$ using Maxstat analysis (cut-off value used for Cdc20 is 11.679 and for Cdh1 8.319). Data were analysed through genomicscape (http://genomicscape.com). $\mathbf{g}-\mathbf{j}$ Immunohistochemical analysis of Cdc20 expression in primary DLBCL patient samples. Immunohistochemical analysis of Cdc20 expression in a DLBCL patient. A $\times 4$ and $\times 40$ magnification is shown (g). Percentage of Cdc20-positive lymphoma cells was counted in 34 DLBCL patients and plotted against the estimated prognosis according to the R-IPI score of the patients (h). Percentage of Cdc20-positive cells was also plotted against the molecular subtype (i) and double-expresser status (j) in 27 DLBCL patients

prognosis compared with the patients with a good prognosis $(p=$ 0.0042) (Fig. 1h). Consistent with the multivariate COX analysis, neither significant correlation between Cdc20 expression and molecular subtype was found (Fig. 1i; Table 1B) nor doubleexpresser status (Fig. 1j). Together, these data suggest that increased $\mathrm{Cdc} 20$ expression is associated with high-grade lymphoma and Cdc20 thus represents an interesting therapeutic target.
Pharmacological inhibition of the APC/C decreases lymphoma cell viability and induces apoptosis

Next, the APC/C was investigated as a potential target in DLBCL and $\mathrm{MCL}$ using human cell lines. Therefore, the Cdc20 and Cdh1 expression was first determined using gene expression profiling data of a large panel of DLBCL and MCL cell lines. Similar to the primary patient samples, we found a significantly higher Cdc20 mRNA expression in both the DLBCL and MCL cell lines compared with B-cell 
Table 1. COX univariate and multivariate analysis of overall survival in R-CHOP-treated DLBCL $(n=233)$ including Cdc20 gene expression

\begin{tabular}{lll}
\hline A. Univariate analysis & \multicolumn{2}{l}{ Overall survival $(n=233)$} \\
\hline Prognostic variable & HR & $p$-value \\
Cdc20 & 2.43 & 0.008 \\
Age ( $>60$ years) & 2.2 & $<0.0001$ \\
GCB-ABC molecular subgroups & 2.75 & $<0.0001$ \\
IPI & 1.79 & $<0.0001$ \\
& & \\
\hline B. Multivariate analysis & Overall survival $(n=233)$ \\
\hline Prognostic variable & HR & $p$-value \\
Cdc20 & 2.91 & 0.01 \\
Age ( $>60$ years) & 0.79 & $n s$ \\
GCB-ABC molecular subgroups & 4.15 & $<0.0001$ \\
IPI & 1.64 & 0.001 \\
\hline
\end{tabular}

The prognostic factors were tested as a single variable $(A)$ or multi variables (B) using the COX-model. $p$-values and hazard ratios (HR) are shown. ns: not significant at a $5 \%$ threshold

samples and a lower Cdh1 mRNA expression (Supplemental Fig. 1a). These findings were also confirmed both at mRNA and protein levels in a selected panel of DLBCL and MCL cell lines (Supplemental Fig. 1b, c). Next, the effect of the specific APC/C inhibitor proTAME was evaluated on the viability of the selected $M C L$ and DLBCL cell lines. As shown in Fig. 2a, a dose-dependent decrease in viability was observed in all cell lines after $24 \mathrm{~h}$ of treatment. Among the MCL cell lines, the Rec- 1 cells were found the least sensitive to proTAME (IC-50 of 19.2 $\mu \mathrm{M})$, while the Jeko- 1 cells were the most sensitive with an IC-50 of $\sim 5.2 \mu \mathrm{M}$. Most of the DLBCL cell lines have an IC-50 value around 6 $\mu \mathrm{M}$, except for the SU-DHL-6 cells which were the most sensitive with an IC-50 of $2.5 \mu \mathrm{M}$ (Fig. 2b). This decreased viability was also confirmed by performing cell counting (Supplemental Fig. 1d). The effect of APC/C inhibition was also determined on apoptosis by using an Annexin $V / 7^{\prime}-A A D$ staining. A significant increase in apoptotic cells was observed in all cell lines (Fig. 2c). Moreover, this induced apoptosis seems to be caspase- 3 mediated, as evidenced by a significant increase in the percentage of active caspase-3-positive cells after APC/C inhibition (Fig. 2d). Similar effects on viability and apoptosis were observed after $48 \mathrm{~h}$ of treatment (Supplemental Fig. 1e-h). Finally, the effect of pharmacological inhibition of the APC/ $C$ was tested on primary patient cells obtained from four $M C L$ and three DLBCL patients. As shown in Fig. $2 \mathrm{e}, \mathrm{f}$, a dose-dependent reduction in viability was observed in all patient samples after $72 \mathrm{~h}$ of proTAME treatment. Patient characteristics can be found in Supplemental Table 1.

APC/C inhibition results in a metaphase arrest

To determine the underlying mechanisms of action, the effect of proTAME on the activity of the co-activators was assessed by determining the protein levels of the APC/C-Cdc20 substrate cyclin $B 1$ and APC/C-Cdh1 substrate Skp2. Cells were synchronised using a double thymidine block and released into proTAME treatment. Western blot analysis demonstrated that cyclin B1 protein levels increased during mitotic arrest in all MCL and DLBCL human cell lines used, as indicated by phosphorylation of the APC/C subunit APC3 (also known as Cdc27). ${ }^{34}$ Skp2 protein levels remained mostly unchanged, except in the ABC-DLBCL cell line (Fig. 3a). In contrast, in untreated synchronised cells, neither significant accumulation of cyclin B1 or Skp2 levels was observed, nor was there an increase in phosphorylation of APC3 (Supplemental Fig. 2). Together, these data indicate that the induced mitotic arrest is proTAME-dependent, as well as the effects seen on cyclin B1 and Skp2 protein levels. As the
$\mathrm{APC} / \mathrm{C}$ is an important protein complex involved in the metaphase-anaphase transition during mitosis, the effect on cell cycle progression was investigated. The mitotic arrest was confirmed by cell cycle analysis using a propidium iodide flow-cytometry staining (Fig. 3b). To further validate these findings, a May-Grünwald Giemsa staining was performed and cells in the metaphase were quantified (Supplemental Fig. 3). A significant increase in the percentage of cells in the metaphase after APC/C inhibition was observed (Fig. 3C). These findings on synchronised cells demonstrate that proTAME treatment induced metaphase arrest in DLBCL and MCL cells.

Induced apoptosis is mediated by phosphorylation of $\mathrm{Bcl}-2$ and $\mathrm{BCl}-\mathrm{xL}$

A prolonged metaphase is known to delay the activation of Cdk1, which can then phosphorylate/inactivate several anti-apoptotic proteins from the $\mathrm{BCl}-2$ family, thus resulting in the activation of the intrinsic apoptotic pathway. ${ }^{35}$ The effect of proTAME treatment on the expression and phosphorylation of $\mathrm{Mcl}-1, \mathrm{BCl}-2$ and $\mathrm{BCl}-\mathrm{xL}$ was evaluated during mitotic arrest by western blot. No clear difference in the total $\mathrm{BCl}-2, \mathrm{BCl}-\mathrm{xL}$ and $\mathrm{Mcl}-1$ protein was observed. Nevertheless, consistent with the prolonged activation of $\mathrm{Cdk} 1$, an increase in phosphorylation of $\mathrm{BCl}-2$ and $\mathrm{BCl}-\mathrm{xL}$ was observed during the mitotic arrest (Fig. 4a). In contrast, neither a difference was observed in the cleaved $\mathrm{Mcl}-1$ protein levels during a mitotic arrest nor in the Cdc4 (also known as FBW7) protein levels. Again, no changes in phosphorylation of $\mathrm{Bcl}-2$ or $\mathrm{Bcl}-\mathrm{xL}$ were observed in the untreated synchronised cells (Supplemental Fig. 2). Together, these data suggest that APC/C targeting induces caspase-mediated apoptosis by inactivating the anti-apoptotic protein $\mathrm{BCl}-2$ and $\mathrm{BCl}-\mathrm{xL}$. Based on this, we next hypothesised that proTAME might sensitise the DLBCL and MCL cell lines to a $\mathrm{BH} 3$ mimetic. To investigate this hypothesis, DLBCL and $M C L$ cell lines were treated with proTAME and the selective Bcl-2 inhibitor ABT199. In all three lymphoma subtypes, an increased percentage of apoptotic cells was observed in the cells treated with proTAME and ABT-199 compared with both single agents (Fig. 4b).

ProTAME enhances the anti-lymphoma activity of the Cdc20/Cdh1 inhibitor apcin and the standard-of-care agent doxorubicin A previous study showed that simultaneous disruption of different protein interactions of the APC/C might be a more effective tool for inactivating this complex. ${ }^{36}$ Therefore, proTAME was combined with a Cdc20/Cdh1 inhibitor, namely apcin, which prevents the coactivator-substrate interaction. Different concentrations (IC-10, IC-30 and IC-50) of both agents were used to test the combinatory effect (Supplemental Table 2). The combination of proTAME and apcin significantly increased the effect of both single agents in all human cell lines (Fig. 5a), with the MCL cell line being the most sensitive. Moreover, this combinatory effect was found to be synergistic in all cell lines, with combination indexes well below 1 for the relevant concentrations. Next, to investigate the effect of APC/C inhibition on the activity of clinically relevant drugs, we performed combination studies with the standard-of-care agents doxorubicin and rituximab. ProTAME treatment was found to significantly and synergistically sensitise lymphoma cells of all the B cell NHL subtypes tested to doxorubicin-mediated cell death, especially at higher concentrations (Fig. 5b). Of interest, the MCL cell line Jeko-1 again seemed more sensitive to the combination than the DLBCL cell lines SU-DHL- 6 and U2932. In contrast, rituximab showed only very minor cytotoxicity in vitro, and this was not further enhanced when combining rituximab with proTAME (Fig. 5c).

\section{DISCUSSION}

Even with recent improvements in the treatment of lymphoma patients, no difference is detected in the overall survival of $\mathrm{MCL}$ patients $^{37}$ and there is still a poor outcome for $30-40 \%$ of the 
a

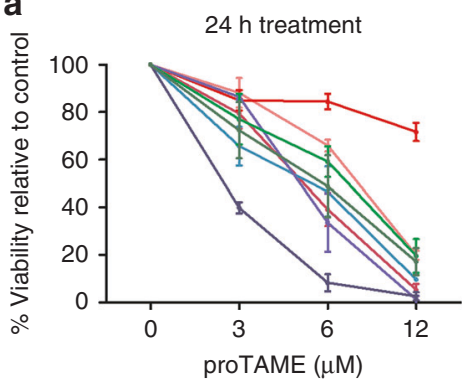

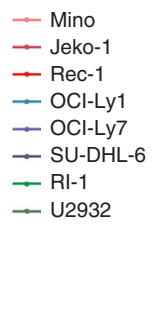

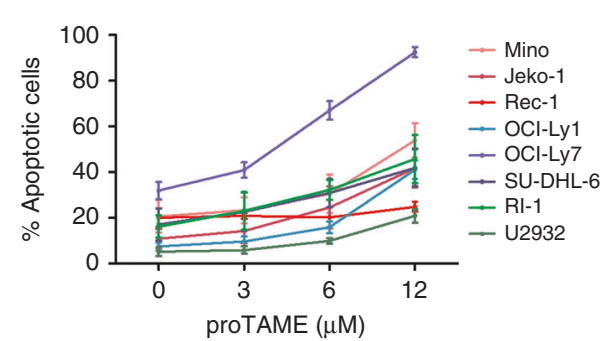

b

\begin{tabular}{|c|c|c|c|c|c|c|c|c|}
\hline $\begin{array}{c}\text { Cell } \\
\text { line }\end{array}$ & Mino & Jeko-1 & Rec-1 & $\begin{array}{c}\text { OCl- } \\
\text { Ly1 }\end{array}$ & $\begin{array}{c}\text { OCI- } \\
\text { Ly7 }\end{array}$ & $\begin{array}{c}\text { SU- } \\
\text { DHL-6 }\end{array}$ & RI-1 & U2932 \\
\hline $\begin{array}{c}\text { IC-50 } \\
(\mu \mathrm{M})\end{array}$ & 7.8 & 5.2 & 19.2 & 5.1 & 5.2 & 2.5 & 6.9 & 5.7 \\
\hline
\end{tabular}

d
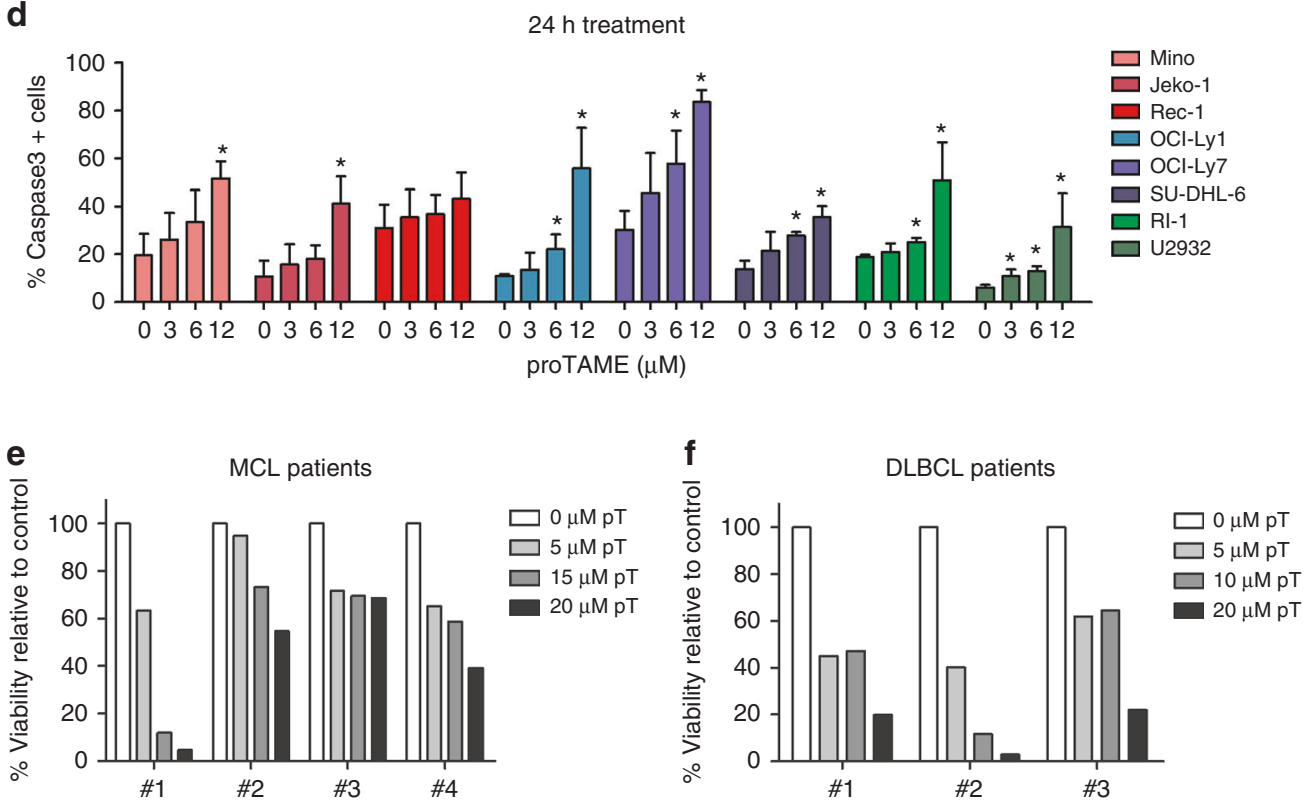

Fig. $2 \mathrm{APC} / \mathrm{C}$ inhibition reduces $\mathrm{DLBCL}$ and $\mathrm{MCL}$ viability and induces caspase-3-mediated apoptosis. $\mathbf{a}$, $\mathbf{b}$ Effect of proTAME treatment on cell viability. MCL and DLBCL cell lines were treated for $24 \mathrm{~h}$ with proTAME $(3,6$ and $12 \mu \mathrm{M})$ and the effect on viability was determined using a CellTiter-Glo assay (a). The results are shown as \% viability relative to control. The IC-50 values for each cell line were calculated using Prism (b). c, $\mathbf{d}$ Effect of proTAME treatment on apoptosis. The effect of proTAME treatment on apoptosis (c) and percentage of active caspase-3- positive cells (d) was determined after $24 \mathrm{~h}$, using an Annexin V/7'-AAD staining, followed by flow-cytometric analysis, and active caspase-3 staining followed by flow-cytometric analysis. The percentage of apoptotic cells are the sum of the percentage of Annexin V and Annexin V/ $7^{\prime}-A A D$ positive cells. The results shown in graphs $\mathbf{a}, \mathbf{c}$ and $\mathbf{d}$ are the mean $\pm \mathrm{SD}$ of three independent experiments. ${ }^{*} p<0.05,{ }^{* *} p<0.01$ and ${ }^{* * *} p<$ 0.001 . e, $\mathbf{f}$ The effect of proTAME treatment on primary patient samples. The viability of the proTAME-treated MCL (e) and DLBCL (f) patient samples was determined after $24 \mathrm{~h}$. The results are shown as \% viability relative to control

DLBCL patients. ${ }^{3}$ The high proliferation rate of these lymphoma cells provides the rationale to use selective antimitotic drugs as a treatment option. ${ }^{37,38}$ In this study, the mitotic exit regulator APC/ $C$ is identified as a novel, promising target in $A B C-D L B C L$, GCB-DLBCL and MCL. From literature, it is known that the activity of the APC/C depends on the interaction with the APC/C coactivators. Changes in the expression/activation of one or both of these co-activators subsequently result in alterations in the APC/C activity. ${ }^{39}$ Thus, to investigate the involvement of the APC/C in DLBCL and $M C L$ disease, we investigated the expression of APC/C co-activators $\mathrm{Cdc} 20$ and $\mathrm{Cdh} 1$ using publicly available gene expression profiling data from DLBCL and $M C L$ patients. We demonstrated that the APC/C co-activator Cdc20 is significantly overexpressed in DLBCL patients and in aggressive $M C L$ patients compared with indolent MCL. Moreover, high Cdc20 gene expression was correlated with a poor survival outcome in the DLBCL patients. Consistent with our results, high Cdc20 gene expression was repeatedly shown to be associated with poor prognosis in various other cancer types, including breast cancer, multiple myeloma, non-small-cell lung cancer, etc. ${ }^{15-21}$ In contrast, there is still some controversy about the role of Cdh1 in tumorigenesis. Cdh1 is generally considered to be a tumour suppressor. ${ }^{11}$ However, two studies showed that loss of Cdh1 function induces cell death and might be essential for tumour development. Moreover, a recent study observed increased Cdh1 expression in primary MM cells. ${ }^{27,40,41}$ In our study, neither a significant difference in Cdh1 mRNA expression was detected in both DLBCL and $\mathrm{MCL}$ patients nor was there a significant 
a
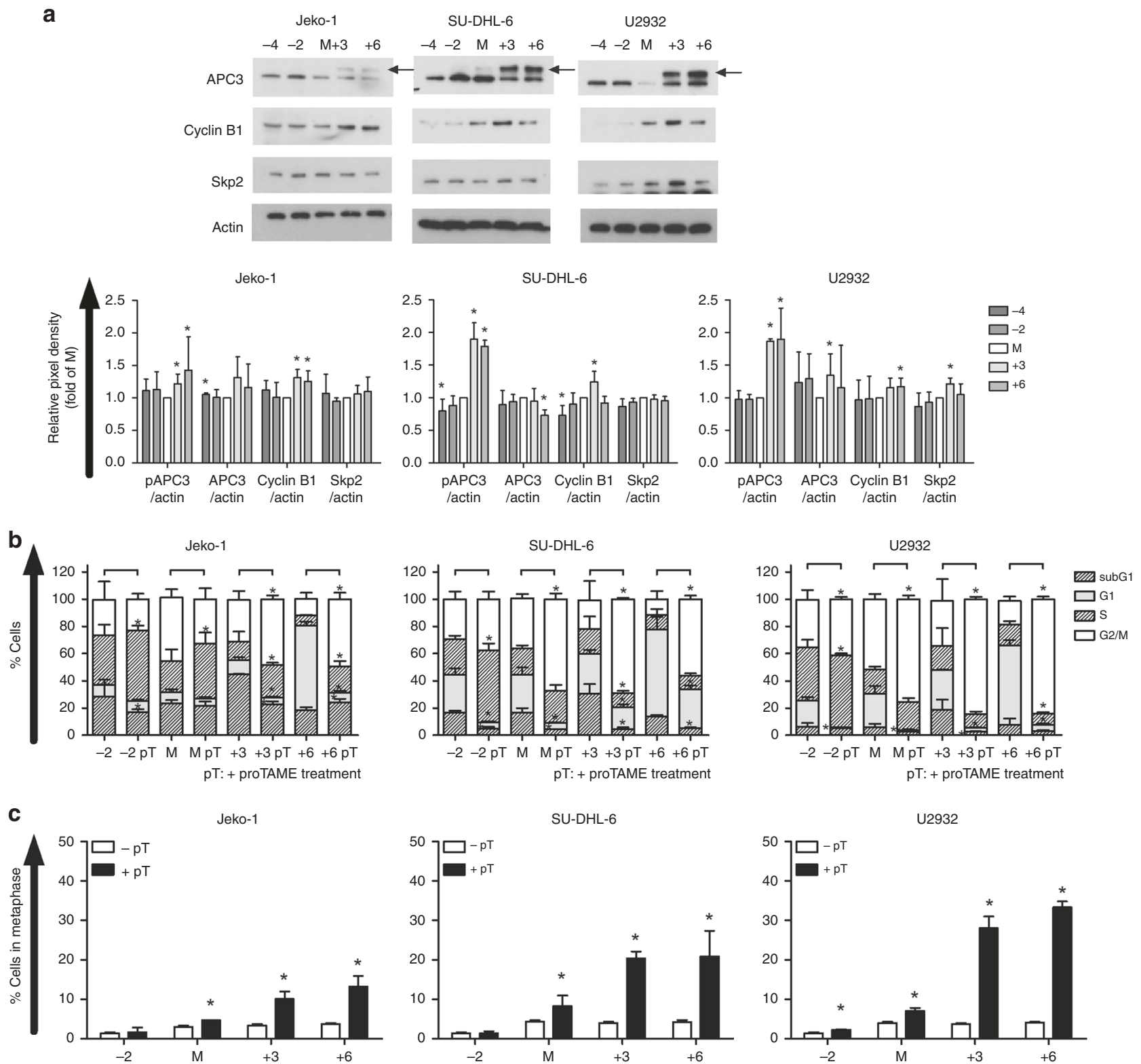

Fig. 3 Pharmacological inhibition of the APC/C with proTAME results in a metaphase arrest. a Effect of proTAME treatment on the substrates of the APC/C co-activators Cdc20 and Cdh1. Expression of cyclin B1 and Skp2 was determined at different timepoints in the MCL and DLBCL cell lines after synchronisation and release into proTAME treatment $(3 \mu \mathrm{M}$ in SU-DHL- 6 and $6 \mu \mathrm{M}$ in Jeko- 1 and U2932). APC3 and $\beta$-actin were used as indicators of mitotic arrest and loading control. Arrows show the phosphorylated APC 3 . One experiment representative of three is shown (the results shown are from the same experiment as those shown in Fig. 4). Normalisation was performed with Image $J$ and quantification relative to the $\mathrm{M}$ condition is shown. Bars represent the mean \pm SD of three independent experiments. $\mathbf{b}$, $\mathbf{c}$ Effect of proTAME treatment on cell cycle progression. The effect on cell cycle progression was determined using PI staining on cell lines treated with $3 \mu \mathrm{M}$ (SUDHL-6) or $6 \mu \mathrm{M}$ (Jeko-1 and U2932) proTAME for the indicated timepoints after synchronisation (b). To further examine the effect on mitosis, cytospins of these treated cells were stained with May-Grünwald Giemsa. For each sample, $3 \times 100$ cells were counted per cytospin. Quantification of the percentage of cells in the metaphase is shown (c). The results shown in each graph are the mean \pm SD of three independent experiments. ${ }^{*} p<0.05$ and ${ }^{* *} p<0.01 . \mathrm{M}=$ mitosis, $-4=4 \mathrm{~h}$ before mitosis, $-2=2 \mathrm{~h}$ before mitosis, $+3=3 \mathrm{~h}$ after mitosis and $+6=6 \mathrm{~h}$ after mitosis

correlation between expression levels and survival. Importantly, aggressive B-cell malignancies are a diverse group of lymphomas, including many variants of $\mathrm{DLBCL}$ and $\mathrm{MCL}$. There are enormous differences in clinical behaviour and molecular mechanisms, as well as response to treatment. Consequently, identification of new prognostic markers is of utmost importance to achieve tailored therapy. ${ }^{42}$ The Cox analysis in our study revealed that Cdc20 is an independent prognostic factor in DLBCL and Cdc20 protein expression seems to be linked to a poor prognosis in DLBCL patients. Importantly, the prognostic value in DLBCL seems to be irrespective of the molecular subtype and double expressers. Based on these findings, Cdc20 could be a potential new, relevant marker for poor prognosis in DLBCL patients. However, further validation on protein level in a prospective study in larger patient cohorts remains warranted. Together, our data indicate that Cdc20 is involved in DLBCL and MCL pathogenesis, thus supporting the preclinical testing of $\mathrm{APC} / \mathrm{C}$ targeting in these aggressive $\mathrm{B}$ cell NHLs.

The therapeutic potential of targeting the APC/C, either by blocking the activation of the complex or the co-activators, has 

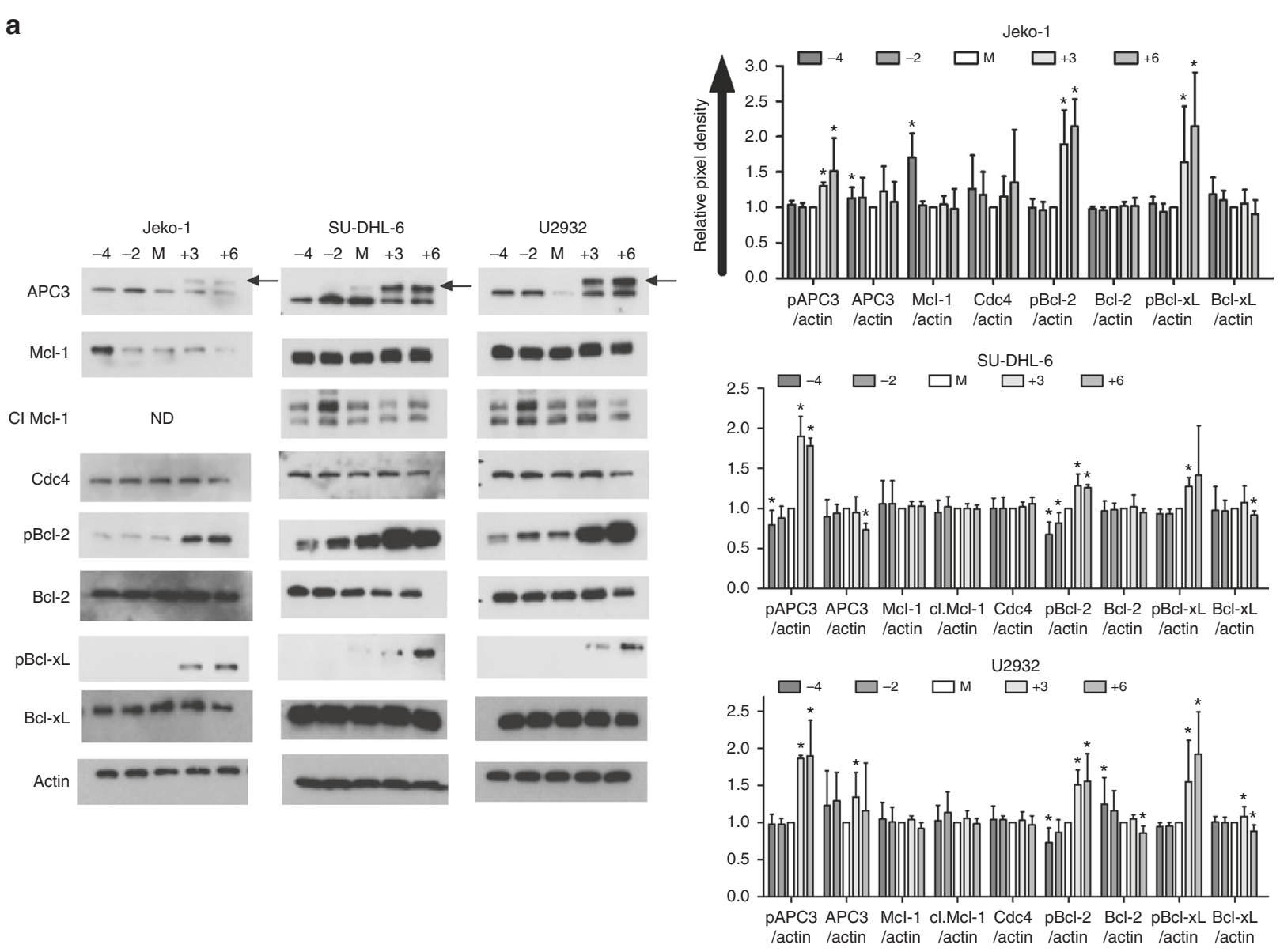

b

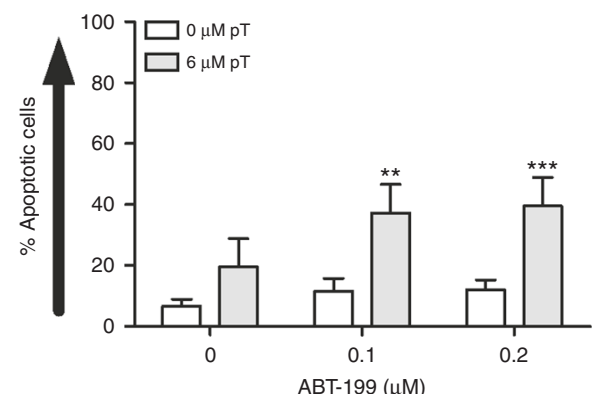

SU-DHL-6

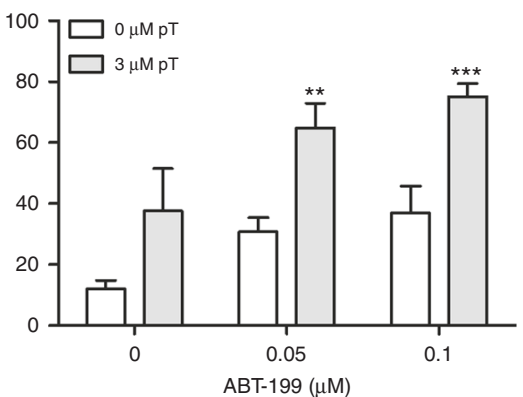

U2932

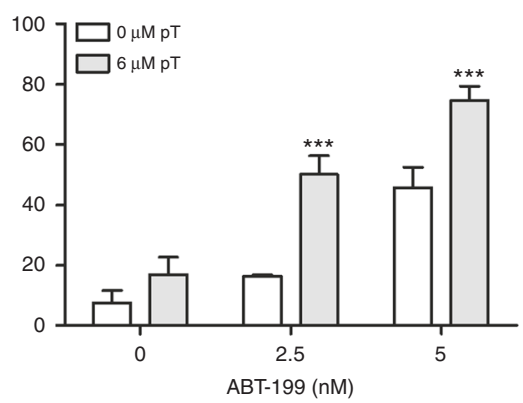

Fig. 4 proTAME inactivates the anti-apoptotic proteins $\mathrm{BCl}-2$ and $\mathrm{BCl}-\mathrm{xL}$ and sensitises $\mathrm{MCL}$ and $\mathrm{DLBCL}$ cells to venetoclax. a Effect of proTAME treatment on the expression and phosphorylation of $\mathrm{Bcl}-2, \mathrm{Bcl}-\mathrm{xL}$ and $\mathrm{Mcl}-1$. The expression of $\mathrm{Mcl}-1, \mathrm{Cdc} 4, \mathrm{Bcl}-2$ and $\mathrm{Bcl}-\mathrm{xL}$ protein and phosphorylation of $\mathrm{BCl}-2$ and $\mathrm{BCl}-\mathrm{xL}$ was determined at different timepoints in the synchronised $\mathrm{MCL}$ and DLBCL cell lines after proTAME treatment $(3 \mu \mathrm{M}$ in SU-DHL-6 and $6 \mu \mathrm{M}$ in Jeko-1 and U2932) using western blot. APC3 and $\beta$-actin were used as indicators of mitotic arrest and loading control. Arrows show the phosphorylated APC3. One experiment representative of three is shown (the results shown are from the same experiment as those shown in Fig. 3). Normalisation was performed with Image $\mathrm{J}$ and quantification relative to the $\mathrm{M}$ condition is shown. Bars represent the mean $\pm S D$ of three independent experiments. $M=$ mitosis, $-4=4 \mathrm{~h}$ before mitosis, $-2=2 \mathrm{~h}$ before mitosis, $+3=3 \mathrm{~h}$ after mitosis and $+6=6 \mathrm{~h}$ after mitosis, ND $=$ not detected. $\mathbf{b}$ The anti-lymphoma effect of APC/C targeting in combination with the BH3 mimetic ABT-199. Apoptosis of proTAME (pT) and ABT-199-treated Jeko-1, SU-DHL-6 and U2932 cells was determined after $48 \mathrm{~h}$ using Annexin V/7'AAD staining followed by flow-cytometric analysis. The sum of the percentage of Annexin V and Annexin V/7'-AAD-positive cells are shown. The results shown in each graph are the mean \pm SD of four independent experiments. Each combination was compared with both single agents. ${ }^{* *} p<0.01$ and ${ }^{* * *} p<0.001$

been examined in cancer using proTAME, ${ }^{15,26,27}$ withaferin $A_{1}^{43}$ NAHA (a hydroxamic acid derivative) ${ }^{44}$ and apcin. ${ }^{45}$ The small molecule proTAME is the only true/selective APC/C inhibitor known so far. ${ }^{26}$ ProTAME has already been described to cause a prolonged metaphase in cancer cells. ${ }^{15,26}$ Consistent with these observations, synchronised $\mathrm{DLBCL}$ and $\mathrm{MCL}$ cells released into proTAME treatment were found to accumulate in the G2/M phase.
Western blot analysis of these cells demonstrated an increase in cyclin B1 protein levels during this mitotic arrest, while Skp2 levels are not or only slightly affected. The induced G2/M-phase arrest was further investigated, and a significant increase in the percentage of cells in the metaphase was observed. It is known that cells in a prolonged metaphase can either undergo cell death or re-enter the cell cycle to exit mitosis. Cdc20 is believed to be a 
a

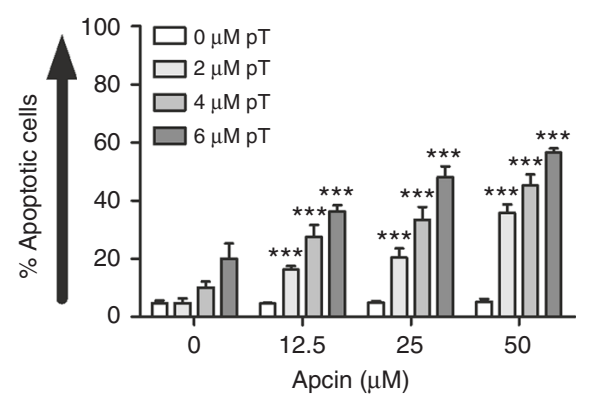

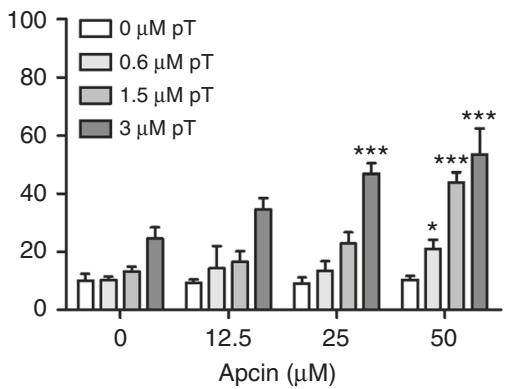

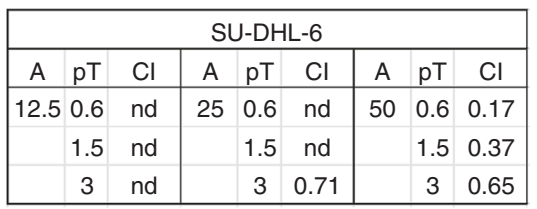

SU-DHL-6
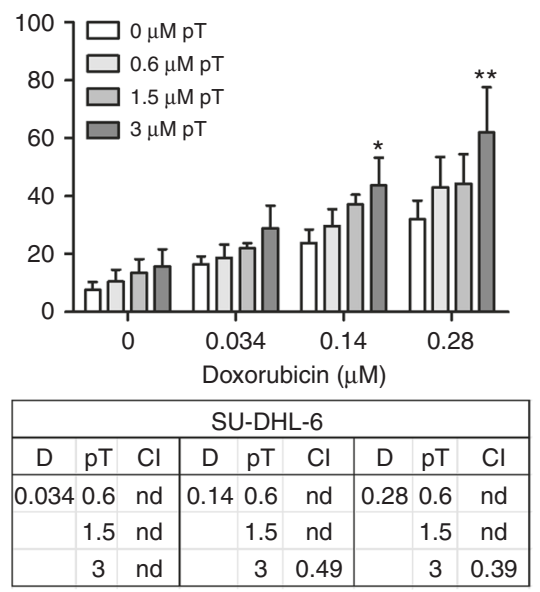

SU-DHL-6

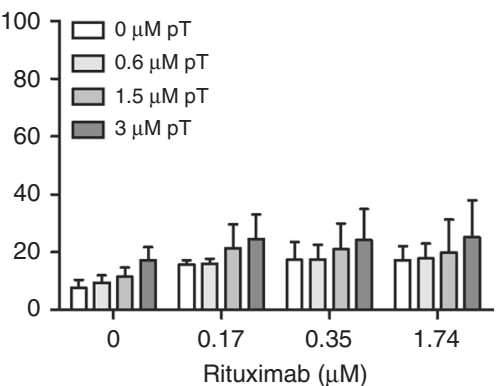

U2932

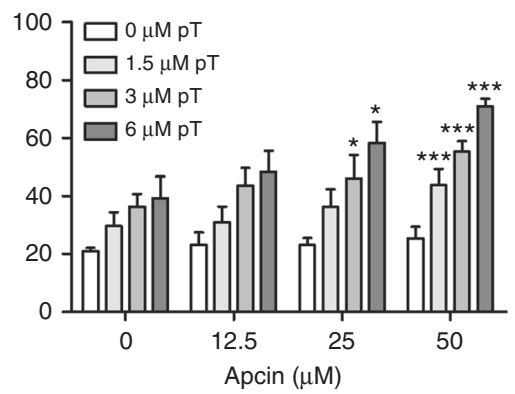

\begin{tabular}{|rcc|ccc|ccc|}
\hline \multicolumn{10}{|c|}{ U2932 } \\
\hline A & pT & Cl & A & pT & Cl & A & pT & Cl \\
\hline 12.5 & 1.5 & nd & 25 & 1.5 & nd & 50 & 1.5 & 0.33 \\
& 3 & nd & & 3 & 0.56 & & 3 & 0.36 \\
\hline 6 & nd & & 6 & 0.62 & & 6 & 0.44 \\
\hline
\end{tabular}

U2932

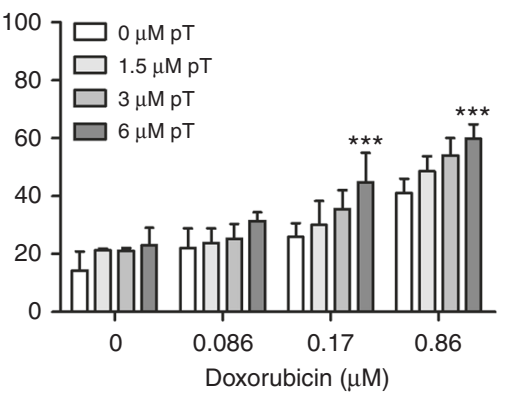

\begin{tabular}{|c|c|c|c|c|c|c|c|c|}
\hline \multicolumn{9}{|c|}{ U2932 } \\
\hline D & pT & $\mathrm{Cl}$ & D & pT & $\mathrm{Cl}$ & D & pT & $\mathrm{Cl}$ \\
\hline 0.086 & 1.5 & nd & 0.17 & 1.5 & nd & 0.86 & 1.5 & nd \\
\hline & 3 & nd & & 3 & nd & & 3 & nd \\
\hline & 6 & nd & & 6 & 0.43 & & 6 & 0.46 \\
\hline
\end{tabular}

U2932

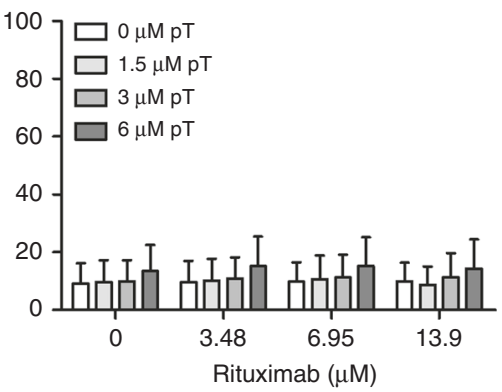

Fig. $5 \mathrm{APC} / \mathrm{C}$ inhibition enhances the anti-lymphoma activity of the Cdc20/Cdh1 inhibitor apcin and the clinically relevant agent doxorubicin. a-c The anti-lymphoma effect of APC/C targeting in combination with apcin, doxorubicin or rituximab. Apoptosis of proTAME (pT) and/or apcin (a), doxorubicin (b) or rituximab (c) treated with Jeko-1, SU-DHL-6 and U2932 cells was determined after $48 \mathrm{~h}$ using Annexin V/7'-AAD staining followed by flow-cytometric analysis. The sum of the percentage of Annexin $V$ and Annexin V/7'-AAD-positive cells are shown. The results shown in each graph are the mean \pm SD of four independent experiments. ${ }^{*} p<0.05,{ }^{* *} p<0.01$ and ${ }^{* * *} p<0.001$ when compared with both single agents. Combination index $(\mathrm{Cl})$ values were calculated for the different drug concentrations by the Chou and Thalalay method using CompuSyn 1.0 software (nd: not determined, $\mathrm{Cl} \leq 1$ : synergistic)

critical factor in this decision-making. When Cdc20 levels increase during the metaphase, cyclin B1 will be degraded, resulting in Cdk1 inactivation and mitotic exit. ${ }^{46}$ On the other hand, a prolonged activation of the cyclin B1-Cdk1 complex occurs when cells are arrested in the metaphase, resulting in the phosphorylation and inactivation of $\mathrm{BCl}-2$ and $\mathrm{BCl}-\mathrm{xL}$, and subsequent activation of the intrinsic apoptotic pathway. ${ }^{35,47,48}$ Consistent with the above, the proTAME-induced prolonged metaphase in
DLBCL and MCL cells is accompanied by a dose-dependent decrease in viability and increase in caspase-mediated apoptosis. Western blot analysis confirmed the phosphorylation of both Bcl-2 and $\mathrm{Bcl}-\mathrm{xL}$. In contrast, $\mathrm{Mcl}-1$ and $\mathrm{Cdc} 4$ levels remained mostly unchanged during the mitotic arrest, indicating that the Cdc4-Mcl-1 axis is most likely not involved in proTAME-induced cell death. These findings provide evidence that APC/C targeting using proTAME induces a metaphase arrest in DLBCL and $\mathrm{MCL}$ 
cells, resulting in the accumulation of cyclin B1 and a prolonged activation of Cdk1, followed by phosphorylation of Bcl-2 and Bcl$\mathrm{xL}$ and eventually cell death.

To further ensure that cells will not re-enter the cell cycle after the metaphase arrest induced by proTAME, combination studies were performed. Previously, we and others demonstrated that inactivation of the APC/C is more effective when multiple protein interactions are simultaneously disrupted using proTAME and apcin. ${ }^{15,36}$ ProTAME blocks the interaction between the APC/C and its co-activators, while apcin inhibits the interaction between the co-activators and their substrates. ${ }^{36}$ This combination resulted in a synergistic anti-lymphoma effect in all cell lines. Other studies in myeloma also showed a combinatory effect with the antimyeloma agents melphalan, vincristine, etoposide and the topoisomerase II inhibitor doxorubicin. ${ }^{15,27}$ Topoisomerase Ila is identified as a Cdh1 substrate and both proTAME and knockdown of Cdh1 significantly enhance the sensitivity of cancers cells to topoisomerase II inhibitors. ${ }^{49}$ Consistent with these findings, proTAME was also found to sensitise all lymphoma cell lines tested to doxorubicin. Combining proTAME with rituximab did not demonstrate any additional effect. This could be explained by the minor effects that rituximab alone had on the different cell lines in vitro. Consistently with our data, others also observed no or only minor effects when using rituximab as monotherapy in DLBCL cell lines. ${ }^{50}$ The anti-lymphoma effect of proTAME combined with a novel agent in clinical development in DLBCL and $\mathrm{MCL}$, namely the selective $\mathrm{BCl}-2$ inhibitor venetoclax, was also tested. ProTAME strongly and significantly potentiated the antilymphoma activity of venetoclax in all cell lines tested, even in the highly sensitive ABC-DLBCL cell line U2932.

To further validate the therapeutic potential of targeting of APC/C in $\mathrm{DLBCL}$ and $\mathrm{MCL}$, we acknowledge that in vivo studies are required. Unfortunately, when it comes to the in vivo use of proTAME, there are some concerns. ProTAME has not yet been optimised for in vivo studies, since this prodrug is activated intracellularly by esterases and the active form (TAME) is not cell permeable. ${ }^{26}$ These enzymes can be present in the bloodstream, thus limiting the in vivo use of proTAME. A possible solution could be to pack (pro)TAME in a liposomal vesicle, such as the vesicle used for liposomal doxorubicin in the treatment of aggressive B-cell lymphoma. ${ }^{51}$ Another general concern is that inhibition of the APC/C will most likely affect all dividing cell types. To avoid these off-target effects, liposomal (pro)TAME could be altered, so that it can only be recognised by CD20+B cells. This would give the advantage of specifically targeting the lymphoma cells like rituximab.

In conclusion, this study demonstrates that high Cdc20 expression correlates with a poor prognosis in the aggressive B-cell malignancy DLBCL. We also provide evidence that APC/C targeting in DLBCL and MCL cells results in a prolonged metaphase followed by apoptosis and sensitises the lymphoma cells to the clinically relevant agents doxorubicin and venetoclax. Together, this study suggests that the $\mathrm{APC} / \mathrm{C}$ is a potential new target in these cancer types, making it a promising target for further preclinical research.

\section{ACKNOWLEDGEMENTS}

The authors would like to thank Catarina Amato, Carine Seynaeve, Lotte Jacobs, Sofie Seghers and Pascal Verhavert for their expert technical assistance.

\section{AUTHOR CONTRIBUTIONS}

A.M., E.V.V., K.V. and E.D.B. conceived and designed the experiments. A.M., K.M., H.D.R., E.D.S., P.V., V.S., J.D., S.F., K.D.V and E.D.B. performed the experiments and analysed the data. A.M. and E.D.B. wrote the paper. A.M., K.M., H.D.R., E.D.S., P.V., V.S., J.D., S.F., K.D.V., E.M., F.O., M.S., J.M., K.V., E.V.V. and E.D.B. provided crucial suggestions, revised the paper and approved the final version. K.V. and E.D.B. supervised the study.

\section{ADDITIONAL INFORMATION}

Supplementary information is available for this paper at https://doi.org/10.1038/ s41416-019-0471-0.

Competing interests: The authors declare no competing interests.

Ethics approval and consent to participate: The study protocol was approved by the ethics committee of the university hospital UZ Brussel and informed consent was obtained from all subjects (B.U.N. 143201836541).

Funding: This work was sponsored by the Fonds voor Wetenschappelijk Onderzoek Vlaanderen, Stichting tegen Kanker, Kom Op tegen Kanker, SRP-VUB and Wetenschappelijk Fonds Willy Gepts (UZ Brussel). Ken Maes, Kim De Veirman and Sylvia Faict are (post)doctoral fellows of FWO-VI.

Consent for publication: Not applicable.

Data availability: The datasets supporting the conclusions are included within the article (and its additional files).

Note: This work is published under the standard license to publish agreement. After 12 months, the work will become freely available and the license terms will switch to a Creative Commons Attribution 4.0 International (CC BY 4.0).

Publisher's note: Springer Nature remains neutral with regard to jurisdictional claims in published maps and institutional affiliations.

\section{REFERENCES}

1. Cummin, T. \& Johnson, P. Lymphoma: turning biology into cures. Clin. Med (North. II). 16, s125-9 (2016).

2. Fakhri, B. \& Hahl, B. Current and emerging treatment options for mantle cell lymphoma. Ther. Adv. Hematol. 8, 223-234 (2017).

3. Miyazaki, K. Treatment of diffuse large b-cell lymphoma. J. Clin. Exp. Hematop. 56, 79-88 (2016).

4. Shankland, K. R., Armitage, J. O. \& Hancock, B. W. Non-Hodgkin lymphoma. Lancet 380, 848-857 (2012).

5. Tangutur, A. D., Kumar, D., Krishna, K. V. \& Kantevari, S. Microtubule targeting agents as cancer chemotherapeutics: an overview of molecular hybrids as stabilizing and destabilizing agents. Curr. Top. Med Chem. 17, 2523-2537 (2017).

6. U.S. National Institutes of Health-ClinicalTrials.gov. www.clinicaltrials.gov.

7. Kelly, K., Shea, T., Goy, A., Berdeja, J., Reeder, C., McDonagh, K. et al. Phase 1 study of MLN8237-investigational aurora A kinase inhibitor-in relapsed/refractory multiple myeloma, non-Hodgkin lymphoma and chronic lymphocytic leukemia. Invest New Drugs 32, 489-499 (2014).

8. Friedberg, J., Mahadevan, D., Jung, J., Persky, D. O., Lossos, I. S., Danaee, H. et al. Phase 2 trial of alisertib (MLN8237), an investigational, potent inhibitor of aurora A kinase (AAK), in patients (pts) with aggressive B- and T-cell non-hodgkin lymphoma (NHL). Blood 118, 95 (2011).

9. Dent, S., Gelmon, K., Chi, K., Jonker, D., Wainman, N., Capier, C. et al. NCIC CTG IND.181: phase 1 study of AT9283 given as a weekly $24 \mathrm{~h}$ infusion in advanced malignancies. Invest New Drugs 31, 1522-1529 (2013).

10. Manchado, E., Guillamot, M. \& Malumbres, M. Killing cells by targeting mitosis. Cell Death Differ. 19, 369-377 (2012).

11. Zhou, Z., He, M., Shah, A. A. \& Wan, Y. Insights into APC/C: from cellular function to diseases and therapeutics. Cell Div. 11, 9 (2016).

12. Penas, C., Ramachandran, V. \& Ayad, N. G. The APC/C ubiquitin ligase: from cell biology to tumorigenesis. Front Oncol. 1, 1-20 (2012).

13. Wang, L., Zhang, J., Wan, L., Zhou, X., Wang, Z. \& Wei, W. Targeting Cdc20 as a novel cancer therapeutic strategy. Pharm. Ther. 151, 141-151 (2015).

14. Wang, Q., Moyret-Lalle, C., Couzon, F., Surbiquet-Clippe, C., Saurin, J., Lorca, T. et al. Alterations of anaphase-promoting complex genes in human colon cancer cells. Oncogene 22, 1486-1490 (2003).

15. Lub, S., Maes, A., Maes, K., De Veirman, K., De Bruyne, E., Menu, E. et al. Inhibiting the anaphase promoting complex/cyclosome induces a metaphase arrest and cell death in multiple myeloma cells. Oncotarget 7, 4062-4076 (2016).

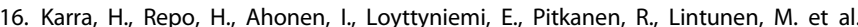
Cdc20 and securin overexpression predicts short-term breast cancer survival. Br. J. Cancer 110, 2905-2913 (2014). 
17. Kato, T., Diego, Y., Aragaki, M., Ishikawa, K., Sato, M. \& Kaji, M. Overexpression of $\mathrm{CDC} 20$ predicts poor prognosis in primary non-small cell lung cancer patients. $J$. Surg. Oncol. 106, 423-430 (2012).

18. Wu, W., Hu, K., Wang, D., Zeng, Z., Zhang, D., Chen, D. et al. Cdc20 overexpression predicts a poor prognosis for patients with colorectal cancer. J. Transl. Med. 11, 142 (2013).

19. Li, J., Gao, J., Du, J., Huang, Z. \& Wei, L. Increased CDC20 expression is associated with development and progression of hepatocellular carcinoma. Int. J. Oncol. 45, 1547-1555 (2014).

20. Ding, Z., Wu, H., Zhang, J., Huang, G. \& Ji, D. Expression characteristics of CDC20 in gastric cancer and its correlation with poor prognosis. Int. J. Clin. Exp. Pathol. 7, 722-727 (2014).

21. Chang, D., Ma, Y., Ji, B., Liu, Y., Hwu, P., Abbruzzese, J. et al. Increased CDC20 expression is associated with pancreatic ductal adenocercinoma differentiation and prognosis. J. Hematol. Oncol. 5, 15 (2012).

22. Kidokoro, T., Tanikawa, C., Furukawa, Y., Katagiri, T., Nakamura, Y. \& Matsuda, K. Cdc20, a potential cancer therapeutic target, is negatively regulated by $\mathrm{p} 53$. Oncogene 27, 1562-1571 (2008).

23. Manchado, E., Eguren, M. \& Malumbres, M. The anaphase-promoting complex/ cyclosome (APC/C): cell-cycle-dependent and -independent functions. Biochem Soc. Trans. 38, 65-71 (2010).

24. Fujita, T., Liu, W., Doihara, H. \& Wan, Y. Regulation of Skp2-p27 axis by the Cdh1/ anaphase-promoting complex pathway in colorectal tumorigenesis. Am. J. Pathol. 173, 217-228 (2008)

25. Fujita, T., Liu, W., Doihara, H., Date, H. \& Wan, Y. Dissection of the APCCdh1-Skp2 cascade in breast cancer. Clin. Cancer Res. 14, 1966-1975 (2008).

26. Zeng, X., Sigoillot, F., Gaur, S., Choi, S., Pfaff, K. L., Oh, D.-C. et al. Pharmacologic inhibition of the anaphase-promoting complex induces a spindle checkpointdependent mitotic arrest in the absence of spindle damage. Cancer Cell. 18, 382-395 (2010)

27. Crawford, L. J., Anderson, G., Johnston, C. K. \& Irvine, A. E. Identification of the APC/C co-factor FZR1 as a novel therapeutic target for multiple myeloma. Oncotarget 7, 70481-70493 (2016).

28. Cardesa-Salzmann, T., Coloma, L., Gutierrez, G., Chan, W., Weisenburger, D., Climent, F. et al. High microvessel density determines a poor outcome in patients with diffuse large B-cell lymphoma treated with rituximab plus chemotherapy. Haematologica 96, 996-1001 (2011).

29. Lenz, G., Wright, G., Dave, S., Xiao, W., Powell, J., Zhao, H. et al. Stromal gene signatures in large-B-cell lymphomas. N. Engl. J. Med2. 359, 2313-2323 (2008).

30. Dybkaer, K., Bogsted, M., Falgreen, S., Bodker, J., Kjeldsen, M., Schmitz, A. et al. Diffuse large B-cell lymphoma classification system that associates normal B-cell subset phenotypes with prognosis. J. Clin. Oncol. 33, 1379-1388 (2015).

31. Fernandez, V., Salamero, O., Espinet, B., Solé, F., Royo, C., Navarro, A. et al. Genomic and gene expression profiling defines indolent forms of mantle cell lymphoma. Cancer Res2. 70, 1408-1418 (2010).

32. Barretina, J., Caponigro, G., Stransky, N., Venkatesan, K., Margolin, A., Kim, S. et al. The cancer cell line encyclopedia enables predictive modelling of anticancer drug sensitivity. Nature 483, 603-607 (2012).

33. Szablewski, V., Bret, C., Kassambara, A., Devin, J., Cartron, G., Costes-Martineau, V. et al. An epigenetic regulator-related score (EpiScore) predicts survival in patients with diffuse large $B$ cell lymphoma and identifies patients who may benefit from epigenetic therapy. Oncotarget 9, 19079-19099 (2018).

34. King, R., Peters, J., Tugendreich, S., Rolfe, M., Hieter, P. \& Kirschner, M. A $20 \mathrm{~S}$ complex containing $\mathrm{CDC} 27$ and $\mathrm{CDC} 16$ catalyzes the mitosis-specific conjugation of ubiquitin to cyclin B. Cell 81, 279-288 (1995).
35. Terrano, D., Upreti, M. \& Chambers, T. Cyclin-dependent kinase 1-mediated BCl$\mathrm{xL} / \mathrm{BCl}-2$ phosphorylation acts as a functional link coupling mitotic arrest and apoptosis. Mol. Cell Biol. 30, 640-656 (2010).

36. Sackton, K. L., Dimova, N., Zeng, X., Tian, W., Zhang, M., Sackton, T. B. et al. Synergistic blockade of mitotic exit by two chemical inhibitors of the APC/C. Nature 514, 646-649 (2014).

37. Vose, J. M. Mantle cell lymphoma: 2017 update on diagnosis, risk-stratification, and clinical management. Am. J. Hematol. 92, 806-813 (2017).

38. Miller, T. P., Grogan, T. M., Dahlberg, S., Spier, C. M., Braziel, R. M., Banks, P. M. et al. Prognostic significance of the Ki-67 - associated proliferative antigen in aggressive non-hodgkin's lymphomas: a prospective southwest oncology group trial. Blood 83, 1460-1466 (1994).

39. Zhang, J., Wan, L., Dai, X., Sun, Y. \& Wei, W. Functional characterization of anaphase promoting complex/cyclosome (APC/C) E3 ubiquitin ligases in tumorigenesis. Biochim Biophys. Acta 1845, 277-293 (2014).

40. Eguren, M., Porlan, E., Manchado, E., Garcia-Higuera, I., Canamero, M., Farinas, I. et al. The APC/C cofactor $\mathrm{Cdh} 1$ prevents replicative stress and p53-dependent cell death in neuronal progenitors. Nat Commun. 4, (2013). https://doi.org/ 10.1038/ncomms3880.

41. Ishizawa, J., Kuninaka, S., Sugihara, E., Naoe, H., Kobayashi, Y., CHiyoda, T. et al. The cell cycle regulator $\mathrm{Cdh} 1$ controls the pool sizes of hematopoietic stem cells and mature lineage progenitors by protecting from genotoxic stress. Cancer Sci. 102, 967-974 (2011).

42. Said, J. Aggressive B-cell lymphomas: how many categories do we need? Mod. Pathol. 1(Suppl), S4, (2013).

43. Das, T., Roy, K., Chakrabarti, T., Mukhopadhyay, S. \& Roychoudhury, S. Withaferin A modulates the Spindle assembly checkpoint by degradation of Mad2-Cdc20 complex in colorectal cancer cell lines. Biochem Pharmacol. 91, 31-39 (2014)

44. Jiang, J., Thyagarajan-Sahu, A., Krchnak, V., Jedinak, A., Sandusky, G. E. \& Sliva, D. NAHA, a novel hydroxamic acid-derivative, inhibits growth and angiogenesis of breast cancer in vitro and in vivo. PLOS ONE 7, e34283 (2012).

45. Gao, Y., Zhang, B., Wang, Y. \& Shang, G. Cdc20 inhibitor apcin inhibits the growth and invasion of osteosarcoma cells. Oncol. Rep. 40, 841-848 (2018).

46. Nilsson, J. Cdc20 control of cell fate during prolonged mitotic arrest: do Cdc20 protein levels affect cell fate in response to antimitotic compounds. Bioessays 33, 903-909 (2011).

47. Eichhorn, J., Sakurikar, N., Alford, S., Chu, R. \& Chambers, T. Critical role of antiapoptotic $\mathrm{BCl}-2$ protein phosphorylation in mitotic death. Cell Death Dis. 4, e834 (2013).

48. Shitashige, M., Toi, M., Yano, T., Shibata, M., Matsuo, Y. \& Shibasaki, F. Dissociation of $\mathrm{Bax}$ from a $\mathrm{BCl}-2 / \mathrm{Bax}$ heterodimer triggered by phosphorylation of serine 70 of Bcl-2. J. Biochem. 130, 741-748 (2001).

49. Eguren, M., Alvarez-Fernandez, M., Garcia, F., Lopez-Contreras, A., Fujimitsu, K., Yaguchi, $H$. et al. A synthetic lethal interaction between APC/C and topoisomerase poisons uncovered by proteomic screens. Cell Rep. 6, 670-683 (2014).

50. Mahadevan, D., Morales, C., Cooke, L., Manziello, A., Mount, D., Persky, D. et al. Alisertib added to rituximab and vincristine is synthetic lethal and potentially curative in mice with aggressive DLBCL co-overexpressing MYC and BCL2. PLoS ONE 9, e95184 (2014).

51. Cruz, E., Ortiz, J., Garcia-Noblejas, A., Alegre, A. \& Saez, R. Liposomal doxorubicin in aggressive $B$ cell lymphoma has similar efficacy to the conventional formulation: results from a retrospective cohort study. Blood 126, 5106 (2015). 